\title{
Eine differenzierte Betrachtung schafft Transparenz und klärt auf
}

Jürg Nadig

Dr. med. Jürg Nadig

Facharzt FMH für Onkologie und Innere Medizin

Präsident Schweizerische Gesell-

schaft für Medizinische Onkologie SGMO

Bannhaldenstrasse 7

CH-8180 Bülach

Tel. 0448627300

Fax 0448627301

juerg.nadig@hin.ch

\section{Résumé}

Se référant à l'article de Christian Marti publié dans le BMS no 27-28/2006 [1], I'auteur souligne que les prix élevés des médicaments reflètent les effets bénéfiques et non pas les coûts de recherche, élevés eux aussi. Au contraire de la prestation médicale, les prix des médicaments ne baissent pas automatiquement quand le marché s'élargit. Comme il n'existe pas de critères pour en mesurer les effets de manière uniforme, il est difficile de quantifier ces effets au moment de la fixation des prix. De ce fait, I'assurance sociale enregistre des conflits de valeur à l'intersection de la médecine et de la science, alors que les mêmes principes médico-éthiques devraient être appliqués à tous les acteurs de la santé publique. Le système d'indemnisation pourrait en outre influencer certaines décisions thérapeutiques.

Si I'on répartit les coûts de médicaments sur les années de vie gagnées, la thérapie d'une jeune femme à I'herceptine adjuvante ne coûte que la

Wer von uns Ärzten denkt nicht wie Herr Marti? Da zahlt die Sozialversicherung staatlich garantierte Preise für immer teurere Onkologika. Der hohe Preis wird mit dem kleinen Marktsegment und den hohen Entwicklungskosten gerechtfertigt, was aber impliziert, dass mit einer Indikationsausweitung der Preis angepasst, meint gesenkt, werden muss, zumal ja bei den Ärzten der Preis der Leistung auch sinkt, wenn die Menge ausgeweitet wird.

Doch weit gefehlt. Mit der Indikationserweiterung sinkt nicht der Preis, sondern die Gewinne der Pharmafirmen steigen, während Ärzte und Spitäler in der gleichen Sozialversicherung dem Gebot der Kostenneutralität oder dem Globalbudget unterworfen sind, das nur ein moitié des dialyses nécessaires alors que la thérapie adjuvante au moyen d'un inhibiteur de l'aromatase chez une femme de 70 ans présentant un risque minime de rechute atteint plusieurs fois le coût d'une thérapie à l'herceptine.

Comme la recherche développe des procédés thérapeutiques toujours plus efficaces, les coûts de la santé augmenteront. En raison du nombre trop élevé de groupes d'intérêts profitant du complexe médico-industriel, l'élargissement des indications thérapeutiques n'influencera guère les prix tandis que les bénéfices des firmes pharmaceutiques et les primes de caisse-maladie ne manqueront pas de prendre l'ascenseur. Comme les coûts de distribution, le risque lié à l'encaissement et les intérêts du crédit accordé à l'entreprise épuisent la petite marge de bénéfice, on ne pourra au moins pas reprocher à l'oncologue de gagner beaucoup d'argent en prescrivant de l'herceptine.

politisch festgelegtes, nach Dignitäten abgestuftes Einkommen erlaubt.

Vertreter der pharmazeutischen Industrie sehen das anders: Nicht nach den Forschungs-, Entwicklungs- und Vermarktungskosten, sondern nach dem Nutzen des neuen Medikaments richte sich der Preis (so dass der Preis, wenn ich das richtig verstehe, eigentlich sogar noch steigen müsste, wenn die Indikation ausgeweitet wird, weil der Nutzen ja noch zunimmt). Von diesen gegensätzlichen Gesichtspunkten aus lässt sich natürlich trefflich streiten.

Interessanterweise unterliegen aber Gehälter von Ärzten in Spitzenpositionen der Pharmaindustrie nicht mehr der Tarmed-Dignität, obwohl auch sie zu einem wesentlichen Teil im 
oder für das öffentliche Gesundheitswesen tätig sind und den Grossteil des Gewinnes aus dem Sozialversicherungsbereich generieren. Dies erstaunt um so mehr, als die medizinethischen Prinzipien (Respekt der Patientenautonomie, nicht schaden, sondern nützen und Gerechtigkeit) ja nicht nur für Ärzte und Pflegende in der Praxis oder am Krankenbett gelten, sondern für alle Mitarbeiter im Gesundheitswesen, also auch für Politiker, Krankenkassen- und Spitalverwalter sowie die Pharmaindustrie. Nur so wird letztlich ein Umfeld geschaffen, das es erlaubt, am Krankenbett diesen Prinzipien gerecht zu werden.

\section{Kosten pro gewonnenes Lebensjahr vergleichen}

Mehr als eine Million Franken koste ein gewonnenes Leben, wenn Herceptin adjuvant beim Mammakarzinom eingesetzt wird, hat Herr Marti berechnet. Dieser Betrag mag auf den ersten Blick hoch scheinen. Im Vergleich dazu kostet eine Dialyse während 20 Jahren aber etwa gleich viel. Deshalb ist es hilfreicher, die Kosten pro gewonnenes Lebensjahr zu vergleichen: Wird eine 40jährigen Frau mit einem Her2positiven Tumor mit monoklonalen Antikörpern geheilt, belaufen sich die Kosten pro gewonnenes Lebensjahr bei einer Lebenserwartung von 80 Jahren lediglich auf 25000 Franken, was der Hälfte der Dialysekosten pro Lebensjahr entspricht. Anderseits ist aber die scheinbar so teure Herceptintherapie fast zehn Mal günstiger als der adjuvante Einsatz eines Aromatasehemmers bei einer 70jährigen Frau mit einem prognostisch günstigen Mammakarzinom, obwohl Aromatasehemmer pro Monat «nur» 270 Franken kosten und damit weit unter den Kosten liegen, die unsere Gesellschaft angeblich bereit sei, bei Krebspatienten monatlich zu bezahlen.

Sicher schafft eine differenzierte Betrachtung des Kosten-Nutzen-Verhältnisses Transparenz und klärt auf. Dies genügt aber nicht. Inskünftig müssten in den randomisierten (Zulassungs-)Studien auch der Zuwachs an Freiheit oder die Verminderung der Abhängigkeit, die der Patient durch die neue Behandlung erlangt, gemessen werden. Diese Daten, die für die Beurteilung des Nutzens einer Behandlung wichtig wären, fehlen aber in den heutigen Lebensqualitätsund Pharmaökonomiestudien.

Ich nehme an, die Opinion Leader setzten sich bei den Krankenkassen für die Kostenübernahme der adjuvanten Herceptintherapie ein, weil sie von deren Nutzen für die betroffenen Frauen überzeugt waren. Ob dieser Nutzen auch preiswert sei, dies zu beurteilen ist ja nicht Auf- gabe der Ärzte, sondern der Eidgenössischen Leistungskommission und des BAG. Durch die Erfassung der Anzahl adjuvanter Herceptintherapien könnten Krankenkassen und BAG Daten zur Indikationsausweitung sammeln und so auf die Preisgestaltung, meint Preissenkung, Einfluss zu nehmen. Dass die Onkologen den Weg zu einem sinnvollen Einsatz der immer begrenzten Mittel im Gesundheitswesen sogar schon ein Stück weit gegangen sind, zeigt die Zusammenarbeit der Vereinigung der Vertrauensärzte und der Gesellschaft für medizinische Onkologie, die in einer anonymisierten Datenbank die Häufigkeit von Off-Label eingesetzten Medikamenten erfassen will, um so erstmals Daten zur Häufigkeit der Indikationsausweitung zu erhalten. Aber auch wenn sich die Ärzte noch mehr als bisher für den gerechten und sinnvollen Einsatz der begrenzten Mittel einsetzen, die Gesundheitskosten werden weiter steigen, weil immer mehr wirksame und lebensverlängernde Therapien zur Verfügung stehen und eingesetzt werden.

\section{Medizinethische Prinzipien und/oder Mammon?}

Mit seiner Frage nach möglichen finanziellen Beziehungsgeschichten zwischen den Opinion Leader und dem Herceptinhersteller mutmasst Herr Marti, ob es denn neben den medizinethischen Prinzipien nicht auch der schnöde Mammon sein könnte, der die Onkologen, zu denen er sich ja auch zählt, verleitet, Herceptin einzusetzen. Falls Herr Marti Hinweise hat, dass einzelne Onkologen für ihre Intervention bei den Krankenkassen finanzielle Vorteile von der Pharmaindustrie erhielten, müssten diese offengelegt werden. Als praktizierender Onkologe kann ich mich lediglich zu dem von ihm behaupteten Verdienst von 4500-9600 Franken bei der Herceptinabgabe äussern.

Betriebswirtschaftlich berechnet wird die Marge von 9\% zwischen Fabrikabgabepreis und Publikumspreis [2], von den Zinsen des Betriebskredits (51/4\% über 6 Monate), den Distributionskosten (2-3\%), dem Inkassorisiko (1-4\%) und dem Verfallrisiko (0,5-1\%) praktisch aufgebraucht [3], ohne dass die Kosten für Bestellwesen und Lagerbewirtschaftung damit abgegolten sind, ganz zu schweigen vom Risiko einer Rückzahlungsklage der santésuisse wegen unwirtschaftlicher, meint teurer Behandlung. Somit gilt auch hier, dass eine differenzierte Betrachtung von Marge und Gewinn Transparenz schaffen kann und aufklärt.

Bleibt noch die Frage, warum gerade die adjuvante Herceptinbehandlung Herrn Marti bewog, 
zur Feder zu greifen, während er auf die Zulassung der Aromatasehemmer zur adjuvanten Behandlung des Mammakarzinoms nicht reagierte, obwohl die Kosten pro gewonnenes Lebensjahr viel höher sein können als bei der Herceptintherapie.

Der adjuvante Einsatz von Herceptin nützt vorerst der betroffenen Frau, weil nur noch halb so viele Patientinnen Fernmetastasen entwickeln wie in der Kontrollgruppe. Er hilft unbestritten auch der forschenden Pharmaindustrie, die ihre innovativen neuen Therapien dem Arzt am Krankenbett zur Verfügung stellen kann. Von steigenden Aktienkursen profitieren die Pensionskassen, vom steigenden Gewinn die öffentliche Hand. Ärzte mit einem Einzelleistungstarif bringt der Mehraufwand für die zusätzliche Verabreichung von Herceptin mehr Einkünfte, allenfalls auch mehr Gewinn. Krankenkassen überwälzen die steigenden Kosten auf die Prämien, was die Solidarität der gesunden Prämienzahler weiter belastet. Wer, ausser diesen gesunden Prämienzahlern, wird sich da für tiefere Preise einsetzen?

\section{Therapiekosten schwierig prognostizierbar}

Diese neuen teuren Therapien, die nur über eine begrenzte Zeit von einem oder zwei Jahren verabreicht werden, können aber für Ärztenetzwerke, die sich über Kopfpauschalen oder ein Globalbudget finanzieren, zum Problem werden: Kostet die Behandlung einer Patientin mit einem Aromatasehemmer, unabhängig von ihrem Körpergewicht, jährlich etwa 3500 Franken, schwanken die Jahreskosten einer Hercep- tintherapie, abhängig vom Körpergewicht, zwischen 40000 und 100000 Franken. Damit laufen Ärzte in Netzwerken mit Budgetverantwortung Gefahr, dass solche Budgetüberschreitungen ihren Gewinn schmälern, während die Patientinnen Gefahr laufen, dass die Behandlungsstrategie vom Finanzierungssystem beeinflusst werden könnte. Es wäre deshalb interessant, von Herrn Marti zu erfahren, wie das Ärztenetzwerk, in dem er arbeitet, dieses Problem löst.

$\mathrm{Da}$ auch bei den Interessenverhältnissen eine differenzierte Betrachtung Transparenz schaffen und aufklären kann, habe ich, gemäss den ASCOAnforderungen, meine möglichen Interessenkonflikte offengelegt.

\begin{tabular}{|c|c|}
\hline Mögliche Interessenkonflikt & e des Autors \\
\hline Autor & Nadig \\
\hline Anstellung & $\begin{array}{l}\text { Spital Bülach } \\
\text { (Beleg- und Konsiliararzt) }\end{array}$ \\
\hline Konsiliartätigkeit & Spital Bülach \\
\hline Aktien & Apotheke zur Rose \\
\hline Honorare & Spital Bülach \\
\hline Reise-Kongress-Vergütungen & Keine \\
\hline Praxisvergütungssystem & Einzelleistungstarif \\
\hline Kaderposition & Praxisinhaber \\
\hline
\end{tabular}

\section{Referenzen:}

1 Marti C. Die Herceptin-Story - Nutzen, Preise und Profite. Schweiz Ärztezeitung. 2006; 87(27/28):1274-5.

2 www.preise.ch

3 Die Kosten für die Medikamentenbewirtschaftung sind nicht in der TL des Tarmed abgegolten. 